\title{
An Investigation into Transition Rule Sets for Optimum-time Firing Squad Synchronization Algorithms on One-dimensional Cellular Automata
}

\author{
Hiroshi UMEO*, Masaya HISAOKA* and Takashi SOGABE* \\ Osaka Electro-Communication University, Graduate School of Engineering, \\ Faculty of Information Science and Technology, Department of Informatics, \\ 18-8, Hatsu-cho, Neyagawa, Osaka 572-8530, Japan
}

Received May 21, 2002; final version accepted October 6, 2002

\begin{abstract}
The firing squad synchronization problem has been studied extensively for more than forty years, and a rich variety of synchronization algorithms have been proposed. In the present paper, we describe a computer-assisted investigation into state transition tables for which optimum-time synchronization algorithms have been designed. We show that the first transition rule set designed by Waksman [(1966) Inf. Control, 9: 66-78] includes fundamental errors which cause unsuccessful firings and that ninety-three percent of the rules are redundant. In addition, the transition rule sets reported by Balzer [(1967) Inf. Control, 10: 22-42], Gerken [(1987), Diplomarbeit, Institut für Theoretische Informatik, Technische Universität Braunschweig, 502] and Mazoyer [(1987) Theor. Comput. Sci., 50: 183-238] are found to include several redundant rules. We also present herein a survey and a comparison of the quantitative aspects of the optimum-time synchronization algorithms developed thus far for one-dimensional cellular arrays.
\end{abstract}

KEYWORDS: cellular automaton, firing squad synchronization problem, Goto's algorithm, Waksman's algorithm, Balzer's algorithm, Mazoyer's algorithm, Gerken's algorithm

\section{Introduction}

In recent years, cellular automata (CA) have been used increasingly to model real phenomena occurring in fields such as biology, chemistry, ecology, economy, geology, mechanical engineering, medicine, physics, sociology, and traffic management. Cellular automata are considered to be a simple model for complex systems in which an infinite one-dimensional array of finite state machines (cells) updates itself in a synchronous manner according to a uniform local rule. Synchronizing a large scale of cellular automata has been known as the firing squad synchronization problem since its development, and the problem was originally proposed by J. Myhill in order to synchronize all parts of selfreproducing cellular automata [10]. The firing squad synchronization problem has been studied extensively for more than forty years [1-17].

In the present paper, we examine the state transition rule sets for the famous firing squad synchronization algorithms that give a finite-state protocol for synchronizing large-scale cellular automata. We focus on the fundamental synchronization algorithms operating in optimum steps on one-dimensional cellular arrays in which the general is located at one end. The algorithms discussed herein are the eight-state Balzer's algorithm [1], the seven-state Gerken's algorithm [2], the six-state Mazoyer's algorithm [6], the 16-state Waksman's algorithm [17] and a number of revised versions thereof. Specifically, we attempt to answer the following questions:

- First, are all previously presented transition rule sets correct? Do these sets contain redundant rules? If so, what is the exact rule set?

- How do the algorithms compare with each other?

In order to answer these questions, we implement all transition rule sets for the synchronization algorithms mentioned above on a computer and check whether these rule sets yield successful firing configurations at exactly $t=2 n-2$ steps for any array of length $n$ such that $2 \leq n \leq 10000$. In addition, we construct a survey of current optimum-time synchronization algorithms and compare transition rule sets with respect to the number of internal states of each finite state automaton, the number of transition rules realizing the synchronization, and the number of statechanges on the array. With the aid of a computer, the first Waksman's transition rule set is shown to include fundamental errors and the set is shown to contain a considerable number of redundancies. Approximately ninety-three percent of the rules are deleted from the original transition rule set. A number of redundant rules that are not used in the synchronization process are also found in other rule sets. We give the smallest rule set for each algorithm. Finally, we present a comparison of the quantitative aspects of the optimum-time synchronization algorithms, including Goto's and Gerken's algorithms, both having a state-change complexity of $\mathrm{O}(n \log n)$. We indicate that the first-in-the-world optimum-time synchronization algorithm proposed by Goto $[3,4]$ has a state-change complexity of $\mathrm{O}(n \log n)$.

*E-mail: \{umeo,masaya,sogabe\}@umeolab.osakac.ac.jp 


\section{Firing Squad Synchronization Problem}

\subsection{Definition of the Firing Squad Synchronization Problem}

The firing squad synchronization problem is formalized in terms of the model of cellular automata. Figure 1 shows a finite one-dimensional cellular array consisting of $n$ cells, denoted by $\mathrm{C}_{i}$, where $1 \leq i \leq n$. All cells (except the end cells) are identical finite state automata. The array operates in lock-step mode such that the next state of each cell (except the end cells) is determined by both its own present state and the present states of its right and left neighbors. All cells (soldiers), except the left end cell, are initially in the quiescent state at time $t=0$ and have the property whereby the next state of a quiescent cell having quiescent neighbors is the quiescent state. At time $t=0$ the left end cell (general) is in the fire-when-ready state, which is an initiation signal to the array. The firing squad synchronization problem is stated as follows. Given an array of $n$ identical cellular automata, including a general on the left end which is activated at time $t=0$, we want to give the description (state set and next-state function) of the automata so that, at some future time, all of the cells will simultaneously and, for the first time, enter a special firing state. The set of states must be independent of $n$. Without loss of generality, we assume $n \geq 2$. The tricky part of the problem is that the same kind of soldier having a fixed number of states must be synchronized, regardless of the length $n$ of the array.

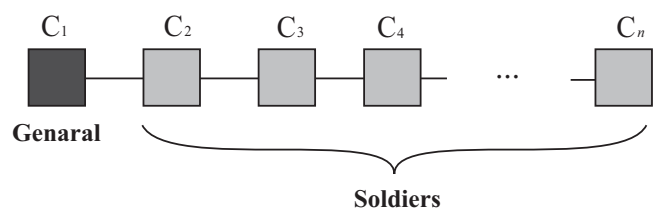

Fig. 1. One-dimensional cellular automaton.

\subsection{A Brief History of the Developments of Optimum-time Firing Squad Synchronization Algorithms}

The problem known as the firing squad synchronization problem was devised in 1957 by Myhill, and first appeared in print in a paper by Moore [10]. This problem has been widely circulated, and has attracted much attention. The firing squad synchronization problem first arose in connection with the need to simultaneously turn on all parts of a selfreproducing machine. The problem was first solved by McCarthy and Minsky who presented a $3 n$-step algorithm. In 1962, the first optimum-time, i.e. $(2 n-2)$-step, synchronization algorithm was presented by Goto [3], with each cell having several thousands of states. Waksman [17] presented a 16-state optimum-time synchronization algorithm. Afterward, Balzer [1] and Gerken [2] developed an eight-state algorithm and a seven-state synchronization algorithm, respectively, thus decreasing the number of states required for the synchronization. In 1987, Mazoyer [6] developed a six-state synchronization algorithm which, at present, is the algorithm having the fewest states.

\subsection{Complexity Measure for Optimum-time Synchronization Algorithms}

\section{- Time}

Any solution to the firing squad synchronization problem can easily be shown to require $(2 n-2)$ steps for firing $n$ cells, since signals on the array can propagate no faster than one cell per step, and the time from the general's instruction until the firing must be at least $2 n-2$. (See Balzer [1], Mazoyer [5,6] and Waksman [17] for a proof.) Thus, we have:

\section{Theorem $1^{[1,5,6,17]}$ Synchronization of $n$ cells in less than $2 n-2$ steps is impossible.}

Theorem $2^{[1-3,6,17]}$ Synchronization of $n$ cells in exactly $2 n-2$ steps is possible.

\section{- Number of internal states}

The following three distinct states: the quiescent state, the general state, and the firing state, are required in order to define any cellular automaton that can solve the firing squad synchronization problem. The boundary state for $\mathrm{C}_{0}$ and $\mathrm{C}_{n+1}$ is not generally counted as an internal state. Balzer [1] showed that no four-state optimum-time solution exists. In addition, there exists no five-state optimum-time solution satisfying the special conditions that Balzer [1] studied. The question that remains is: "What is the minimum number of states for an optimum-time solution of the problem?" At present, that number is five or six.

Theorem $3^{[1]}$ There is no four-state CA that can synchronize $n$ cells. 
Theorem $4^{[1]}$ There is no five-state solution satisfying Balzer's special conditions.

- Number of transition rules

Any $k$-state (excluding the boundary state) transition table for the synchronization has at most $(k-1) k^{2}$ entries in $(k-1)$ matrices of size $k \times k$. The number of transition rules reflects the complexity of synchronization algorithms.

- Number of state changes

Vollmar [16] introduced state-change complexity in order to measure the efficiency of cellular algorithms and showed that $\Omega(n \log n)$ state changes are required for the synchronization of $n$ cells in $(2 n-2)$ steps.

Theorem $5^{[16]} \Omega(n \log n)$ state-change is necessary for synchronizing $n$ cells in $(2 n-2)$ steps.

\section{Transition Rule Sets for Optimum-time Firing Squad Synchronization Algorithms}

\subsection{Waksman's 16-state Algorithm}

In 1966, Waksman [17] proposed a 16-state firing squad synchronization algorithm, which, together with an unpublished algorithm by Goto [3], is referred to as the first-in-the-world optimum-time synchronization algorithm. Waksman [17] devised an efficient way to cause a cell to generate infinite signals at propagating speeds of $1 / 1,1 / 3,1 / 7, \ldots, 1 /\left(2^{k}-1\right)$, where $k$ is any natural number. These signals play an important role in dividing the array into two, four, eight, ..., equal parts synchronously. The end cell in each partition takes a special prefiring state so that when the last partition occurs, all cells have a left and right neighbor in this state. Figure 2 shows the Waksman's timeoptimum firing scheme. Waksman presented a set of transition rules described in terms of a state transition table that is defined on the following state set $\mathcal{D}$ consisting of 16 states such that $\mathscr{D}=\left\{\mathrm{Q}, \mathrm{T}, \mathrm{P}_{0}, \mathrm{P}_{1}, \mathrm{~B}_{0}, \mathrm{~B}_{1}, \mathrm{R}_{0}, \mathrm{R}_{1}, \mathrm{~A}_{000}, \mathrm{~A}_{001}\right.$, $\left.\mathrm{A}_{010}, \mathrm{~A}_{011}, \mathrm{~A}_{100}, \mathrm{~A}_{101}, \mathrm{~A}_{110}, \mathrm{~A}_{111}\right\}$, where $\mathrm{Q}$ is a quiescent state, $\mathrm{T}$ is a firing state, $\mathrm{P}_{0}$ and $\mathrm{P}_{1}$ are prefiring states, $\mathrm{B}_{0}$ and $B_{1}$ are states for signals propagating at various speeds, $R_{0}$ and $R_{1}$ are trigger states which cause the $B_{0}$ and $B_{1}$ states move in the left or right direction and $\mathrm{A}_{i j k}, i, j, k \in\{0,1\}$ are control states which generate the state $\mathrm{R}_{0}$ or $\mathrm{R}_{1}$ either with a unit delay or without any delay. To describe the state transition table in a concise way, Waksman introduced a special symbol $\gamma$ that matches any state in its right and/or left neighbor. Since Waksman's table cannot be used as is on a computer, we first expand the table automatically according to the notation given by Waksman [17]. We thereby obtain a transition table that consists of 3208 rules. We implement these rules and examine the validity of the expanded table on a computer. The experiment revealed the following:

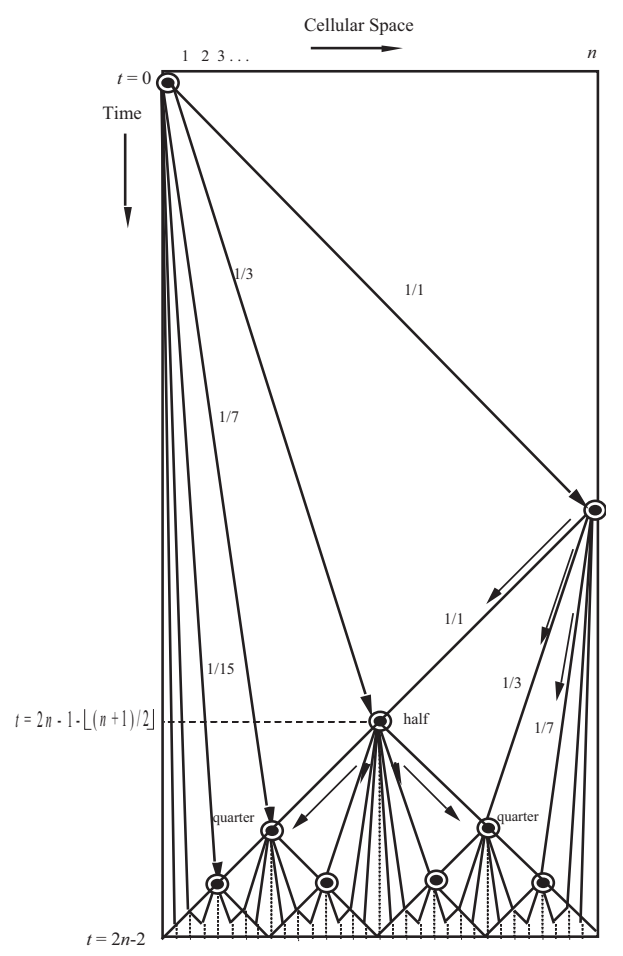

Fig. 2. Time-space diagram for Waksman's optimum-time firing squad synchronization algorithm. 
Observation 1 For any $n$ such that $2 \leq n \leq 2000$, the expanded rule set yields successful firings only in the cases of $n=3,5$ or 6 .

Thus, the firing process fails in most cases.

\subsection{USN Transition Rule Set}

Umeo, Sogabe and Nomura [14] corrected all errors in Waksman's original transition table. In this subsection, we present an overview of the correction procedures described in Umeo, Sogabe and Nomura [14] and give a complete list of the transition rules which yield successful firings for any $n$. Waksman [17] presented a figure (Fig. 2 in Waksman [17]) that illustrates a firing configuration on $n=11$. Even the configuration given by Waksman [17] can not be obtained by our computer simulation. Judging from the original figure in Waksman's paper [17], and from our numerous computer simulations, we can assume that Waksman's figure was obtained by hand simulation. Investigating the figure, Waksman appears to have forgotten to include the following two rules:

$$
\mathrm{R} 1 \# \text { \# BO \#\# PO } \rightarrow \text { R1; R1 \#\# BO \#\# P1 } \rightarrow \text { R1. }
$$

We have added these rules to the original list, and hereafter refer to the rule set as Waksman's original list. Again, we perform the computer simulation based on the rule set consisting of 3210 rules. We observed the following:

Observation 2 For any $n$ such that $2 \leq n \leq 2000$, Waksman's original rule set yields successful firings only for the cases in which $n=3,5,6,11,12,23,24,47,48,95,96,191,192,383,384,767,768,1535$ or 1536.

Thus, a problem remains in the original transition table. Cellular automata researchers have reported that errors are included in the Waksman's transition table. Our computer simulation reveales this to be true, and the original rule set includes fundamental errors. Next, we explore the transition rule set so that the resultant table yields correct firings for any $n \geq 2$. Our investigations on unsuccessful configurations revealed that the signal $\mathrm{A}_{i j k}, i, j, k \in\{0,1\}$ disappears in the cellular space and, as a result, synchronous divisions fail. Based on computer-assisted investigations, we altered five rules in the original list and obtained the rules shown below:

$$
\begin{gathered}
\text { BO\#\# } 2 \# \text { P } 1 \rightarrow \text { A100; B1 \#\# } 2 \# \# \text { PO } \rightarrow \text { A000; PO\#\# } 2 \# \text { \# B } \rightarrow \text { A010; } \\
\text { PO\#\# 2\#\# R1 } \rightarrow \text { A010; P1\#\# Q \#\# BO } \rightarrow \text { A110. }
\end{gathered}
$$

All of these rules involve the transmission of the $\mathrm{A}_{i j k}$ signals. Moreover, we added the following seven rules, which are needed for the above correction:

$$
\begin{aligned}
& \text { PO \#\# 2 \#\# www } \rightarrow \text { P1;A010 \#\# Q \#\# R1 } \rightarrow \text { A011;A011 \#\# Q \#\# R1 } \rightarrow \text { A010; }
\end{aligned}
$$

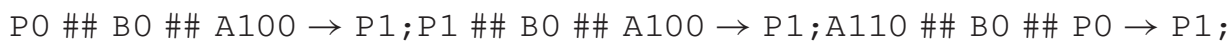

$$
\begin{aligned}
& \text { A110 \#\# BO \#\# P1 } \rightarrow \text { P1. }
\end{aligned}
$$

Overall, the total number of transition rules is 3217. We herein omit the details of the process of exploring the table. The computer simulation based on the new rule set reveals the following:

Observation 3 For any $n$ such that $2 \leq n \leq 10000$, the rule set consisting of 3217 rules yields successful optimumtime firings.

Our computer simulation based on the above list revealed that most of the rules are not used efficiently in the firing process. A histogram-like statistical analysis on the rule set is presented herein and the number of rules valid in the range of $2 \leq n \leq 10000$ is reduced. Figure 3 is our final, complete list, which consists of 202 transition rules. We refer to this list as the USN transition rule set. In our transition table (Fig. 3), for ease of computer simulation, the symbol "www" is used to denote the left and right boundary states.

In our correction, a ninety-three percent reduction in the number of transition rules is realized compared to Waksman's original list. The computer simulation based on the table of Fig. 3 gives the following observation. Computer simulation shows that 202 rules is the smallest set for the Waksman's optimum-time firing squad synchronization. In Fig. 4, we show a configuration on 25 cells.

Observation 4 The set of rules given in Fig. 3 is the smallest transition rule set for Waksman's optimum-time firing squad synchronization algorithm.

\subsection{Balzer's Eight-state Algorithm}

Balzer [1] constructed an eight-state, 182-rule synchronization algorithm and the structure of which is identical to that of Waksman [17]. Our computer examination revealed no errors, however, 17 rules were found to be redundant. In Fig. 5, we give a list of transition rules for Balzer's algorithm together with a configuration on 28 cells. Deleted rules 


\begin{tabular}{|c|c|c|c|c|c|c|c|c|c|c|c|c|c|}
\hline$\cdots$ & $\mathbf{Q}$ & tate -..- & & -..- & B0 Stat & ate -..-- & & & R1 Stat & te & $-\ldots \quad A$ & A000 State & \\
\hline $001 Q$ & $\# \#$ & $\# \# Q \quad->C$ & $\rightarrow Q$ & $062 \mathrm{Q}$ & \#\#BO \# & $\# \#$ & $->\mathrm{BO}$ & 117 Q \# & \#\#R1 \# & $\# \# Q \quad \rightarrow Q$ & $175 \mathrm{Q} \ddagger$ & \#\#A000 \#\#Q $\quad->$ & 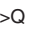 \\
\hline $002 Q$ & $\# \# \mathrm{Q}$ & \#\#BO $\rightarrow$ & $\rightarrow Q$ & $063 \mathrm{Q}$ & \#\#BO & \#\#RO & $\rightarrow \mathrm{R} 0$ & $118 \mathrm{Q} \quad \#$ & \#\#R1 \# & \#\#B0 $\quad->\mathrm{B} 1$ & $176 \mathrm{Q}$ & \#\#A000 \#\#P0 -> & $>\mathrm{BO}$ \\
\hline $003 \mathrm{Q}$ & $\# \#$ & \#\#B1 $\rightarrow$ & $\rightarrow Q$ & $064 \mathrm{Q}$ & \#\#B0 & \#\#PO & $->\mathrm{B} 0$ & 119 Q \# & \#\#R1＃ & \#\#B1 $\quad>$ >B0 & $177 \mathrm{~B} 1$ & \#\#A000 \#\#Q $\quad$-> & $>Q$ \\
\hline $004 \mathrm{Q}$ & $\# \# \mathrm{Q}$ & \#\#RO $\quad$-> & $->\mathrm{R} 0$ & $065 \mathrm{Q}$ & \#\#B0 & \#\#P1 - & $->\mathrm{BO}$ & $120 \mathrm{~B} 1 \mathrm{\#}$ & \#\#R1 \# & $\# \# Q \quad \rightarrow Q$ & $178 \mathrm{~B} 1$ & \#\#A000 \#\#P0 -> & ->BO \\
\hline $005 Q$ & $\# \# \mathrm{Q}$ & \#\#R1 -> & $\rightarrow Q$ & $066 \mathrm{Q}$ & \#\#B0 & \#\#A001 & $1->P 1$ & $121 \mathrm{~B} 1 \mathrm{\#}$ & \#\#R1 \# & \#\#P0 $\quad$->B0 & & & \\
\hline $006 \mathrm{Q}$ & $\# \# \mathrm{Q}$ & \#\#PO $\quad \rightarrow$ & $\rightarrow$ A000 & $067 \mathrm{Q}$ & \#\#BO & \#\#A100 & $0->\mathrm{P} 1$ & $122 \mathrm{~B} 1 \mathrm{\#}$ & \#\#R1 \# & \#\#P1 $\quad$->B0 & $-A_{1} \rightarrow-1$ & A001 State --.-- & \\
\hline $007 Q$ & $\# \# \mathrm{Q}$ & $\# \# \mathrm{P} 1 \quad \rightarrow$ & $->A 100$ & 068 B1 & \#\#B0 & \#\#PO & $->\mathrm{B} 0$ & 123 A101 & 1 \#\#R1 & $\# \# Q \quad->Q$ & & Avor state -...- & \\
\hline $008 \mathrm{Q}$ & $\# \# \mathrm{Q}$ & \#\#A000 - & ->A001 & 069 B1 & \#\#B & \#\#1 & $\rightarrow>\mathrm{BO}$ & $124 \mathrm{~A} 101$ & 1 \#\#R1 & \#\#P1 $\quad$->B0 & $179 \mathrm{Q}$ & \#\#A001 \#\#Q $\quad$-> & $>Q$ \\
\hline $009 \mathrm{Q}$ & $\# \# \mathrm{Q}$ & \#\#A001- & 1 ->A000 & $070 \mathrm{R} 1$ & \#\#B0 & $\# \# \mathrm{Q}$ & $->\mathrm{R} 1$ & -..-- & P0 State & te ------ & $180 Q$ & \#\#A001 \#\#B0 & $\rightarrow Q$ \\
\hline $010 Q$ & $\# \# \mathrm{Q}$ & \#\#A100 - & ->A101 & 071 R1 & \#\#B0 & \#\#PO & $->\mathrm{R} 1$ & $125 \mathrm{Q} \#$ & \#\#PO \# & $\# \# Q \quad->P O$ & $181 \mathrm{~B} 0$ & $\# \# A 001 \# \# Q$ & $\rightarrow Q$ \\
\hline $011 \mathrm{Q}$ & $\# \# \mathrm{Q}$ & \#\#A101 - & 1 ->A100 & 072 R1 & \#\#B0 & \#\#P1 & $\rightarrow \mathrm{R} 1$ & $126 \mathrm{Q} \#$ & \#\#PO \# & \#\#PO $\quad$->PO & $182 \mathrm{~B} 0$ & \#\#A001 \#\#B0 -> & $\rightarrow Q$ \\
\hline $012 \mathrm{Q}$ & $\# \# \mathrm{Q}$ & \#\#A010- & ->RO & $073 \mathrm{PO}$ & \#\#B0 & $\# \#$ Q & $>$ - B0 & $127 \mathrm{Q} \#$ & \#\#РО \# & \#\#www ->PO & & & \\
\hline $013 Q$ & $\# \# \mathrm{Q}$ & \#\#A110 - & $D>Q$ & $074 \mathrm{PO}$ & \#\#B0 & \#\#B1 - & $->\mathrm{B} 0$ & $128 \mathrm{BO}$ \# & \#\#PO & \#\#BO $->$ PO & -..-- A & A100 State ------ & \\
\hline 014 Q & $\# \# \mathrm{Q}$ & \#\#www - & $\rightarrow>Q$ & $075 \mathrm{PO}$ & \#\#B0 & \#\#RO & $->\mathrm{R} 0$ & $129 \mathrm{BO} \#$ & \#\#PO \# & \#\#PO & 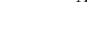 & 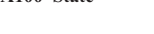 & \\
\hline 015 B0 & $\# \#$ & $\# \# \mathrm{Q} \quad->$ & $>Q$ & $076 \mathrm{PO}$ & \#\#В0 & \#\#PO & $->\mathrm{PO}$ & $130 \mathrm{BO} \#$ & \#\#PO \# & \#\#www ->P0 & $183 \mathrm{Q}$ & \#\#A100 \#\#Q $\quad \rightarrow$ & $>\mathrm{R} 1$ \\
\hline 016 B0 & $\# \# Q$ & \#\#B0 & $\rightarrow Q$ & $077 \mathrm{PO}$ & \#\#В0 & \#\#P1 & $->P 0$ & $131 \mathrm{R} 1 \nRightarrow$ & \#\#PO \# & \#\#R0 $\quad->\mathrm{PO}$ & $184 \mathrm{Q}$ & \#\#A100 \#\#P1 $\quad>$ & $>\mathrm{R} 1$ \\
\hline $017 \mathrm{~B} 0$ & $\# \# Q$ & \#\#R0 $->$ & $->\mathrm{R} 0$ & $078 \mathrm{PO}$ & \#\#B0 & \#\#A100 & 0 ->P1 & $132 \mathrm{R} 1$ & \#\#PO \# & \#\#P0 ->PO & $185 \mathrm{~B} 0$ & \#\#A100 \#\#Q $\quad$-> & $>P 1$ \\
\hline 018 B0 & $\# \# \mathrm{Q}$ & $\# \#$ 1 -> & $\rightarrow$ A100 & 079 P0 & \#\#B0 & \#\#A011 & 1 ->BO & $133 \mathrm{R} 1$ & \#\#PO \# & \#\#Www ->P0 & $186 \mathrm{BO}$ & \#\#A100 \#\#P1 -> & $\rightarrow P 1$ \\
\hline 019 B0 & $\# \# \mathrm{Q}$ & \#\#A000 & ->A001 & $080 \mathrm{P} 1$ & \#\#B0 & $\#$ \# & $->\mathrm{B} 0$ & $134 \mathrm{PO} \#$ & \#\#PO \# & \#\#Q $\quad$->PO & $\left.A_{1}\right)_{2}$ & & \\
\hline 020 B0 & $\# \# \mathrm{Q}$ & \#\#A101 & 1 ->A100 & $081 \mathrm{P1}$ & \#\#B0 & \#\#B1 & $->\mathrm{BO}$ & $135 \mathrm{PO} \#$ & \#\#PO \# & \#\#B0 $\quad->P 0$ & A & Al01 State - & \\
\hline 021 B0 & $\# \#$ & \#\#A010 & $\mathrm{D}>\mathrm{R} 0$ & $082 \mathrm{P} 1$ & \#\#B0 & \#\#R0 & $\rightarrow>\mathrm{R} 0$ & $136 \mathrm{PO} \#$ & \#\#PO \# & \#\#R0 $\quad$->P0 & & \#\#A101 \#\#R1 $\quad->$ & \\
\hline 022 B0 & $\# \# \mathrm{Q}$ & \#\#A110 & $D \rightarrow Q$ & $083 \mathrm{P} 1$ & \#\#B0 & \#\#P0 - & $\rightarrow>P 0$ & $137 \mathrm{PO} \#$ & \#\#PO \# & \#\#PO $\quad$->T & $188 \mathrm{~B} 1$ & \#\#101\#\#R1 -> & $\rightarrow \longrightarrow$ \\
\hline 023 B1 & $\# \# \mathrm{Q}$ & $\# \# Q$ & $>Q$ & $084 \mathrm{P} 1$ & \#\#B0 & \#\#A100 & $0->\mathrm{P} 1$ & $138 \mathrm{PO} \#$ & \#\#PO \# & \#\#1 $\quad->$ T & & AAPIVI & \\
\hline 024 B1 & $\# \#$ & \#\#B1 $->$ & $\rightarrow Q$ & $085 \mathrm{AO}$ & 01 \#\#B0 & \#\#P0 & $->\mathrm{B} 0$ & $139 \mathrm{PO} \#$ & \#\#PO \# & \#\#A010 ->P0 & & A010 State ------ & \\
\hline 025 B1 & $\# \# Q$ & \#\#R0 $\rightarrow>$ & ->R0 & $086 \mathrm{A0}$ & 11 \#\#В0 & $\# \#$ & ->P1 & $140 \mathrm{PO} \#$ & \#\#PO \# & \#\#www $->\top$ & & & \\
\hline 026 B1 & $\# \# Q$ & \#\#R1 -> & $\rightarrow Q$ & 087 A1 & 10 \#\#B0 & $\# \# \mathrm{Q}$ & $->P 1$ & $141 \mathrm{P} 1 \mathrm{\#}$ & \#\#P0 \# & \#\#PO $\quad \rightarrow \mathrm{T}$ & $189 \mathrm{Q}$ & \#\#A010\#\#Q $\quad \rightarrow>$ & $>Q$ \\
\hline 027 B1 & $\# \#$ & \#\#P0 -> & $\rightarrow$ A000 & 088 A1 & 10 \#\#В0 & \#\#P0 & $->\mathrm{P} 1$ & $142 \mathrm{P} 1 \#$ & \#\#PO & \#\#P1 $\quad->\mathrm{T}$ & $190 \mathrm{Q}$ & \#B1 $\rightarrow$ & \\
\hline 028 B1 & $\# \# \mathrm{Q}$ & \#\#A001. & 1 - >A000 & 089 A1 & 10 \#\#В0 & \#\#P1 & ->P1 & $143 \mathrm{P} 1$ & \#\#PO \# & \#\#www ->T & $191 \mathrm{PO}$ & \#\#A010\#\#Q & $>$ BO \\
\hline $\begin{array}{l}\text { O29 B1 } \\
\text { 030 R0 }\end{array}$ & $\begin{array}{l}\# \# Q \\
\# \# Q\end{array}$ & $\begin{array}{l}\text { \#\#A100- } \\
\text { \#\#Q -> }\end{array}$ & $\begin{array}{l}0>\text { - }>\text { A101 } \\
\rightarrow \text { Q }\end{array}$ & $\cdots$ & B1 Stat & te & & $144 \mathrm{~A} 000$ & \#\#PO & \#\#PO $->\mathrm{PO}$ & $192 \mathrm{PO}$ & \#\#A010 \#\#B1 -> & $>\mathrm{BO}$ \\
\hline $031 \mathrm{RO}$ & $\# \# \mathrm{Q}$ & \#\#B1 -> & $\rightarrow Q$ & & Dit siat & (a) & & $145 \mathrm{~A} 000$ & & & & & \\
\hline 032 R0 & $\#$ \#Q & \#\#PO $->$ & $\Rightarrow \mathrm{A} 000$ & $090 \mathrm{Q}$ & \#\#B1 \# & $\#$ \#Q & ->B1 & & $\begin{array}{l}\text { \#\#PO } \\
\text { \#\#PO }\end{array}$ & $\begin{array}{ll}\# \# w w w & ->P 0 \\
\# \# Q & ->P O\end{array}$ & & A011 State -- & \\
\hline 033 R0 & $\#$ \#Q & \#\#A000 & 0 ->A001 & $091 \mathrm{Q}$ & \#\#B1 \# & \#\#BO & $\rightarrow \mathrm{B} 1$ & $\begin{array}{l}147 w w w \\
148 w w w\end{array}$ & \#\#PO & \#\#BO $\quad->P 0$ & $193 \mathrm{Q}$ & \#\#A011 \#\#Q $\quad->$ & $>Q$ \\
\hline $\begin{array}{l}034 \mathrm{RO} \\
\text { 035 R0 }\end{array}$ & $\begin{array}{l}\# \# Q \\
\# \# Q\end{array}$ & $\begin{array}{l}\text { \#\#A001 } \\
\text { \#\#A011. }\end{array}$ & $\begin{array}{l}1>A 000 \\
1 \rightarrow Q \\
1 \rightarrow Q\end{array}$ & $\begin{array}{l}092 \mathrm{Q} \\
093 \mathrm{Q}\end{array}$ & $\begin{array}{l}\text { \#\#1 \# } \\
\text { \#\#B1 \# }\end{array}$ & $\begin{array}{l}\text { \#\#R0 } \\
\text { \#\#R1 }\end{array}$ & $\begin{array}{l}->\mathrm{Q} \\
->\mathrm{B} 1\end{array}$ & 149 www & \#\#PO & \#\#RO $\quad->$ PO & $194 \mathrm{Q}$ & \#\#A011 \#\#B0 -> & $>Q$ \\
\hline $036 \mathrm{R} 1$ & $\begin{array}{l}\text { \#\#Q } \\
\# \# Q\end{array}$ & $\#$ \#Q -> & $\rightarrow \mathrm{R} 1$ & $\begin{array}{l}093 \mathrm{Q} \\
094 \mathrm{Q}\end{array}$ & & \#\#A000 & & 150 www & \#\#PO & \#\#P0 $\quad->\mathrm{T}$ & $195 \mathrm{~B} 0$ & \#\#A011 \#\#Q $\quad$-> & $>Q$ \\
\hline 037 R1 & $\# \# \mathrm{Q}$ & \#\#BO & $->\mathrm{R} 1$ & $095 \mathrm{Q}$ & \#\#B1 \# & \#\#A101 & 1 ->PO & 151 www & \#\#PO & $\begin{array}{lll}\# \# P 1 \quad->T \\
\# \# A 010->P\end{array}$ & $196 \mathrm{~B} 0$ & \#\#A011 \#\#B0 -> & $\rightarrow Q$ \\
\hline $038 \mathrm{R} 1$ & $\# \# \mathrm{Q}$ & \#\#B1 -> & ->R1 & 096 B0 & \#\#B1 & $\# \#$ Q - & ->B1 & & I \#\#P0 & \#\#A010 ->PO & & A110 State ---.-- & \\
\hline 039 P0 & $\# \# \mathrm{Q}$ & $\# \# Q \quad->$ & $\rightarrow A 010$ & 097 B & \#\#1 & \#\#RO & $\rightarrow Q$ & & & & & & \\
\hline 040 P0 & $\# \# \mathrm{Q}$ & \#\#B1 & $->A 010$ & 098 B0 & \#\#B1 & \#\#A000 & 0 ->P0 & $P$ & P1 State & $\begin{array}{ll}\text { ate } & - \\
\# \# Q & ->P 1\end{array}$ & & \#\#A110 \#\#Q $\quad$-> & \\
\hline $041 \mathrm{P} 0$ & $\# \#$ & \#\#R1 -> & ->A010 & 099 B0 & \#\#B1 & \#\#A101 & 1 ->P0 & $153 \mathrm{Q} \quad \#$ & \#\#P1 \# & $\begin{array}{ll}\# \# Q & >P \text { 1 } \\
\# \# P 1 & ->P 1\end{array}$ & 198 Q & \#\#A110 \#\#B0 & $\rightarrow P 1$ \\
\hline $042 \mathrm{P} 0$ & $\# \#$ & \#\#www & ->P1 & $100 \mathrm{RO}$ & \#\#B1 & $\#$ \# - - & $->B 1$ & $154 \mathrm{Q} \#$ & \#\#1 \#\# & $\begin{array}{ll}\# \# P 1 & ->P 1 \\
\# \# w w w & ->P 1\end{array}$ & $199 \mathrm{P} 1$ & \#\#A110\#\#Q - & $\rightarrow$ RO \\
\hline $043 \mathrm{P} 1$ & $\# \# \mathrm{Q}$ & $\# \# \mathrm{Q} \quad \rightarrow$ & $\rightarrow$ A110 & $101 \mathrm{RO}$ & \#\#B1 & \#\#A000 & 0 ->P0 & $155 \mathrm{Q} \quad \#$ & \#\#P1 \#キ & $\begin{array}{l}\text { \#\#www }->\mathrm{P} 1 \\
\text { \#\#B0 }->\mathrm{P} 1\end{array}$ & $200 \mathrm{P} 1$ & \#\#A110 \#\#B0 - & $\rightarrow P 1$ \\
\hline 044 P1 & $\# \#$ & \#\#B0 & $->A 110$ & $102 \mathrm{R} 1$ & \#\#B1 & $\# \# \mathrm{Q}$ & $\rightarrow Q$ & $156 \mathrm{BO} \#$ & \#\#P1 \# & $\begin{array}{ll}\# \# \text { \#0 } & ->\mathrm{P} 1 \\
\# \# P 1 & \rightarrow \mathrm{P} 1\end{array}$ & & & \\
\hline $045 \mathrm{~A} 000$ & 0 \#\#Q & $\# \#$ & $->\mathrm{R} 1$ & $103 \mathrm{R} 1$ & \#\#B1 & \#\#B0 & $\rightarrow Q$ & $157 \mathrm{BO} \#$ & \#\#P1 \# & \#\#P1 $\quad$->P1 & ------ & A111 State & \\
\hline $046 \mathrm{~A} 000$ & 0 \#\#Q & \#\#B0 & $\rightarrow \mathrm{R} 1$ & $104 \mathrm{AO}$ & 10 \#\#B1 & $\# \# \mathrm{Q}$ & $->\mathrm{PO}$ & $158 \mathrm{BO} \#$ & \#\#1 \# & $\#$ \#www ->P1 & & & \\
\hline 047 A001 & 1 \#\#Q & \#\#R1 & $\rightarrow Q$ & $105 \mathrm{AO}$ & 10 \#\#B1 & \#\#B0 & $->\mathrm{PO}$ & $159 \mathrm{R} 1$ & \#\#P1 \# & $\begin{array}{ll}\# \# \mathrm{R} 0 & ->\mathrm{P} 1 \\
\# \text { P } & -\mathrm{P} 1\end{array}$ & 201 Ro & \#\#A111 \#\#Q - & $\rightarrow$ Q \\
\hline 048 A100 & 0 \#\#Q & \#\#Q - & $->Q$ & $106 \mathrm{AO}$ & 10 \#\#B1 & \#\#R1 & $->P 0$ & $160 \mathrm{R} 1$ & \#\#P1 \# & $\begin{array}{lll}\# \# P 1 & ->P 1 \\
\# \# \text { P } & \rightarrow>P 1\end{array}$ & 202 R0 & \#\#A111 \#\#B1 & ->PO \\
\hline 049 A100 & 0 \#\#Q & \#\#BO & $\rightarrow Q$ & $107 \mathrm{~A} 1$ & 11 \#\#B1 & $\# \# \mathrm{Q}$ & $->\mathrm{PO}$ & $161 \mathrm{R} 1$ \# & \#\#P1 \# & $\begin{array}{l}\# \# w w w->P 1 \\
\# \# P 0->T\end{array}$ & & & \\
\hline $050 \mathrm{~A} 010$ & $0 \# \# Q$ & $\# \# \mathrm{Q}$ & ->A011 & $108 \mathrm{~A} 1$ & 11 \#\#B1 & \#\#B0 & $->\mathrm{PO}$ & $162 \mathrm{PO} \quad \#$ & \#\#P1 \# & $\begin{array}{ll}\# \# P 0 & ->T \\
\text { \#\#P1 } & ->T\end{array}$ & & & \\
\hline 051 A010 & $0 \# \#$ & \#\#BO & $->A 011$ & & R0 State & & & $\begin{array}{l}163 \mathrm{PO} \quad \# \\
164 \mathrm{PO} \#\end{array}$ & $\begin{array}{ll}\# \# \text { P1 } & \# \\
\text { \#\#P1 } & \text { \# }\end{array}$ & $\begin{array}{l}\text { \#\#P1 } \quad>T \\
\text { \#\#www } \rightarrow>T\end{array}$ & & & \\
\hline $\begin{array}{l}052 \mathrm{~A} 010 \\
053 \mathrm{~A} 010\end{array}$ & $\begin{array}{l}0 \# \#, \\
0 \# \# Q\end{array}$ & $\begin{array}{l}\text { \#\#R1 } \\
\text { \#\#www }\end{array}$ & $\begin{array}{l}->\mathrm{A} 011 \\
w \rightarrow P 0\end{array}$ & & Ko state & the --..-- & & $165 \mathrm{P} 1 \#$ & \#\#P1 \# & $\# \# Q$ & & & \\
\hline 054 A011 & $1 \# \#$ & $\# \# Q$ & $\rightarrow A 010$ & $109 \mathrm{Q}$ & \#\#RO \# & $\#$ \#Q $\quad>$ & $>Q$ & $166 \mathrm{P} 1 \quad \#$ & \#\#P1 & \#\#B0 $\quad->\mathrm{P} 1$ & & & \\
\hline 055 A011 & 1 \#\#Q & \#\#B1 & $->A 010$ & $110 \mathrm{Q}$ & \#\#R0 \# & \#\#B1 -> & $>Q$ & 167 P1 \# & \#\#P1＃ & \#\#R0 $\quad>$ P1 & & & \\
\hline 056 A011 & 1 \#\#Q & \#\#R1 & $\rightarrow$ A010 & $111 \mathrm{Q}$ & \#\#R0 \# & \#\#A111. & $->Q$ & $168 \mathrm{P} 1 \quad \#$ & \#\#P1 \# & $\begin{array}{lll}\# \# 0 & ->T\end{array}$ & & & \\
\hline 057 A011 & 1 \#\#Q & \#\#www & $w->P 1$ & $112 \mathrm{BO}$ & \#\#R0 \# & $\# \# Q$ & $>$ B1 & $169 \mathrm{P} 1 \quad \#$ & \#\#P1 \# & \#\#P1 $\quad->\top$ & & & \\
\hline 058 A110 & $0 \# \#$ & $\#$ \#Q - & $\Rightarrow \mathrm{A} 111$ & $113 \mathrm{~B} 1$ & \#\#RO \# & $\# \# Q$ & $>$ BO & $170 \mathrm{P} 1 \quad \#$ & \#\#P1＃ & \#\#A110 ->P1 & & & \\
\hline 059 A110 & $0 \# \#$ & \#\#B1 & $->A 111$ & $114 \mathrm{PO}$ & \#\#RO \# & \#\#B1 - & ->BO & $171 \mathrm{P} 1 \quad \#$ & \#\#P1 \# & \#\#www $->T$ & & & \\
\hline 060 A111 & 1 \#\#Q & \#\#Q & ->A110 & $\begin{array}{l}115 \mathrm{P} 1 \\
116 \mathrm{P}\end{array}$ & \#\#R0 \# & \#\#B1 -> & $->\mathrm{B} 0$ & 172 A100 & O \#\#P1 & \#\#P1 $\quad$->P1 & & & \\
\hline 061 A111 & $1 \# \# Q$ & \#\#B0 & $->A 110$ & $116 \mathrm{P} 1$ & \#\#R0 \# & \#\#A111 & 1 ->B0 & $\begin{array}{l}173 \mathrm{~A} 100 \\
174 \mathrm{~A} 100\end{array}$ & $\begin{array}{l}\text { \#\#P1 } \\
\text { \#\#P1 }\end{array}$ & $\begin{array}{l}\text { \#\#A110 }->\mathrm{P} 1 \\
\text { \#\#www }->\mathrm{P} 1\end{array}$ & & & \\
\hline
\end{tabular}

Fig. 3. USN transition table consisting of 202 rules that realize Waksman's synchronization algorithm.

are indicated by shaded squares, where squares containing letters denote rules included in the original list. In the transition table, the symbols "M", "L", "F" and "X" represent the general, quiescent, firing and boundary states, respectively. The state-change complexity of the algorithm is $\mathrm{O}\left(n^{2}\right)$.

\subsection{Gerken's Seven-state Algorithm I}

Gerken [2] reduced the number of states realizing Balzer's algorithm and constructed a seven-state, 118-rule synchronization algorithm. In our computer examination, no errors were found, however, 13 rules were found to be redundant. In Fig. 6, we give a list of the transition rules for Gerken's algorithm together with a configuration on 28 cells. The 13 deleted rules are marked by shaded squares in the table. The symbols " > , “"”, “. ..” and "\#” represent the general, quiescent, firing and boundary states, respectively. The state-change complexity of the algorithm is $\mathrm{O}\left(n^{2}\right)$.

\subsection{Mazoyer's Six-state Algorithm}

Mazoyer [6] proposed a six-state, 120-rule synchronization algorithm, the structure of which differs greatly from the previous algorithms discussed above. Our computer examination revealed no errors and only one redundant rule. In Fig. 7, we give a list of transition rules for Mazoyer's algorithm together with a configuration on 28 cells. In the transition table, the letters "G", "L", "F" and "X" represent the general, quiescent, firing and boundary states, respectively. The state-change complexity of the algorithm is $\mathrm{O}\left(n^{2}\right)$. 


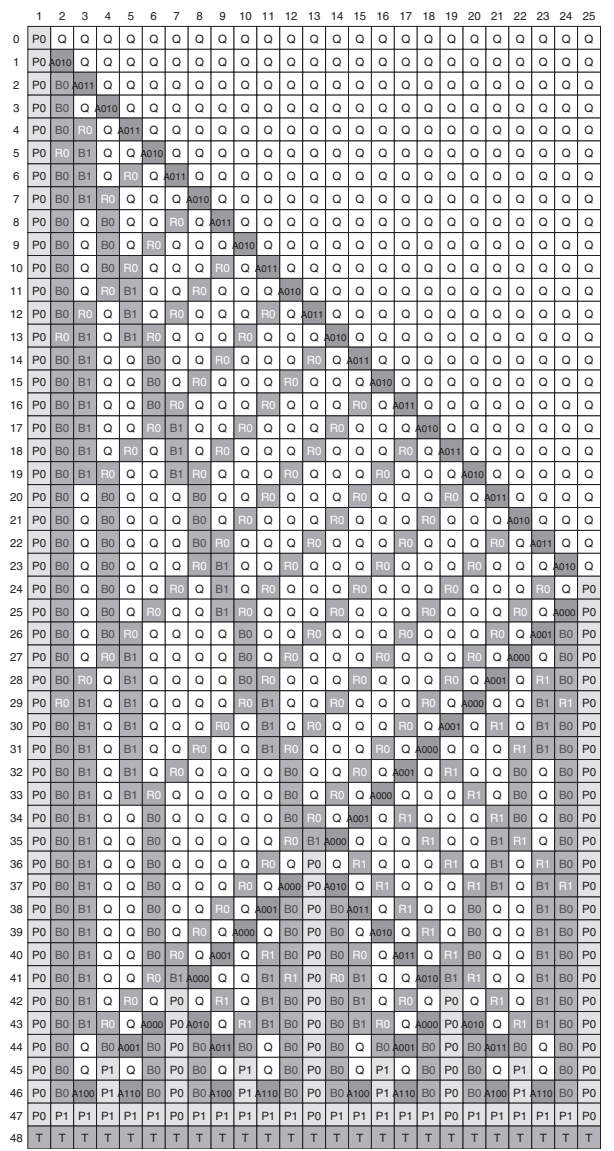

Fig. 4. A configuration of 16-state implementation of Waksman's algorithm on 25 cells.

\subsection{Goto's Algorithm}

The first synchronization algorithm presented by Goto [3] was not published as a journal paper. According to Goto, the original note [3] is now unavailable, and the only existing material that treats the algorithm is Goto [4]. The Goto's study presents one figure (Fig. 3.8 in Goto [4]) demonstrating how the algorithm works on 13 cells with a very short description in Japanese. Umeo [12] reconstructed the algorithm of Goto based on this figure. Mazoyer [8] also reconstructed this algorithm again based on the presentation given by Umeo [12]. The algorithm that Umeo [12] reconstructed is a non-recursive algorithm consisting of a marking phase and a firing phase. In the first phase, by printing a special marker in the cellular space, the entire cellular space is divided into subspaces, the lengths of which increase exponentially with a common ratio of two, that is $2^{j}$, for any integer $j$ such that $1 \leq j \leq\left\lfloor\log _{2} n\right\rfloor-1$. The marking is made from both the left and right ends. In the second phase, each subspace is synchronized using a wellknown conventional $3 n-\mathrm{O}(1)$-step simple synchronization algorithm. A time-space diagram of the reconstructed algorithm is shown in Fig. 8. We note that the state-change complexity of the algorithm is $\mathrm{O}(n \log n)$.

\subsection{Gerken's 155-state Algorithm II}

Gerken [2] constructed two kinds of optimum-time synchronization algorithms. One seven-state algorithm has been discussed in the previous subsection, and the other is a 155-state algorithm having $\mathrm{O}(n \log n)$ state-change complexity. The transition table given in Gerken [2] is described in terms of two-layer construction with 32 states and 347 rules. The table does not operate as is on a single-layer simulator. In order to obtain an exact transition rule set, we expand this transition table into a single-layer format and obtain a 155-state table consisting of 2371 rules. In Fig. 9 we give a configuration on 28 cells.

\subsection{Comparison of Quantitative Aspects of Optimum-time Synchronization Algorithms}

Here, we present a table (Table 1) based on a quantitative comparison of optimum-time synchronization algorithms and their transition tables discussed above with respect to the number of internal states of each finite state automaton, the number of transition rules realizing the synchronization, and the number of state-changes on the array.

\subsection{O(1)-bit vs. 1-bit Communication CA Model}

In the study of cellular automata, the amount of bit-information exchanged at one step between neighboring cells has 

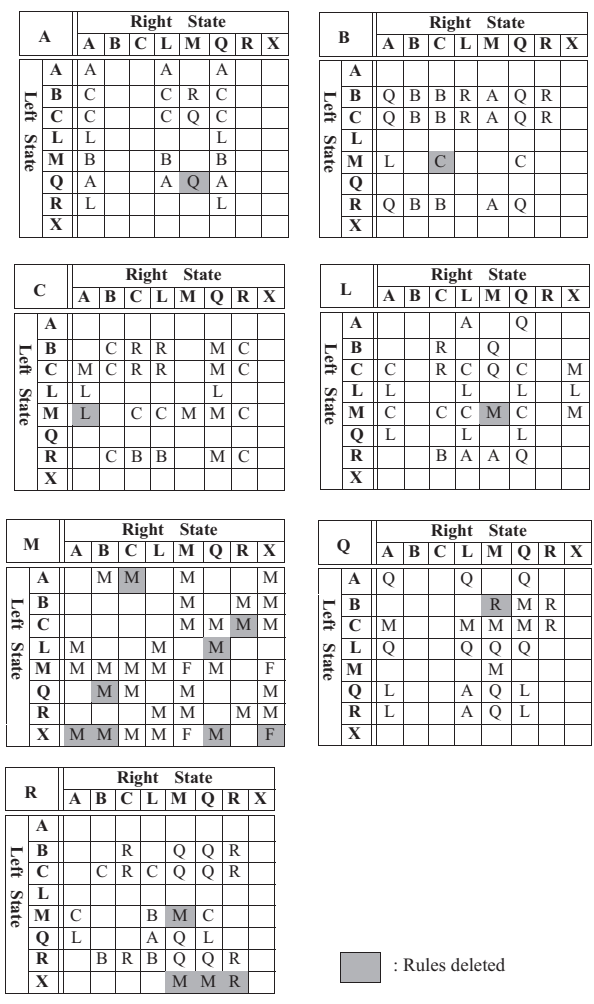

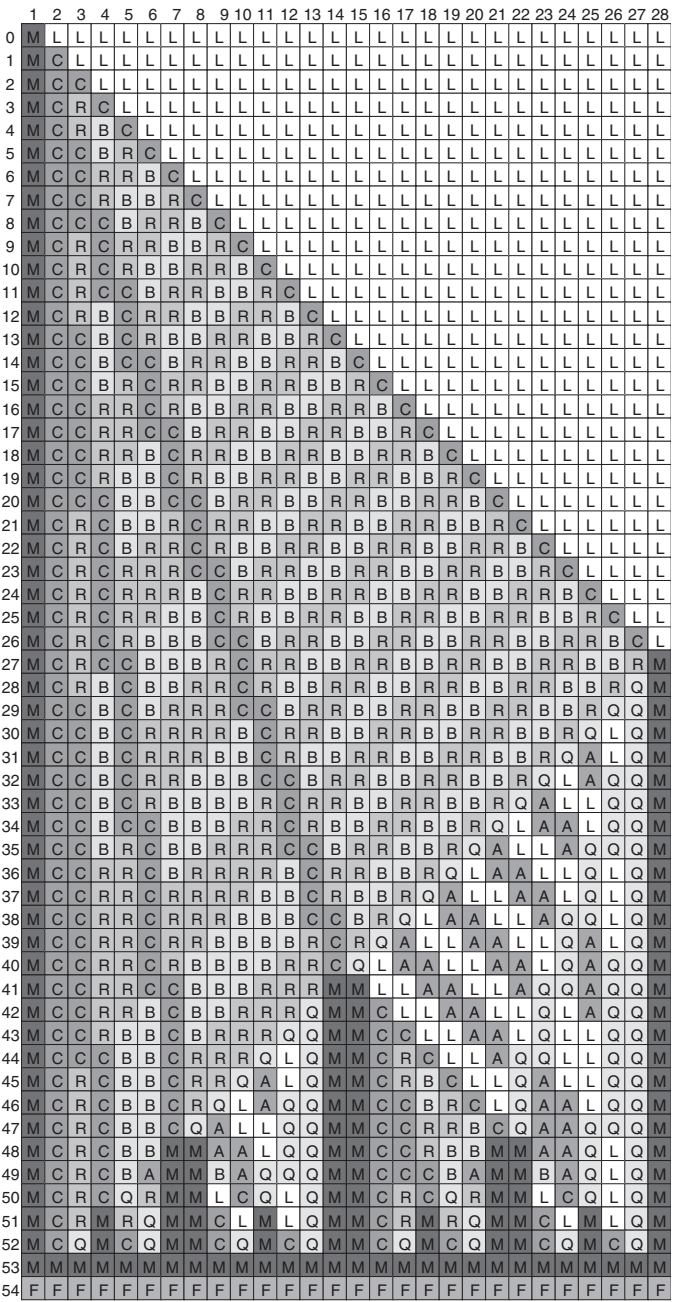

Fig. 5. Transition table for Balzer's algorithm (left) and a configuration of an eight-state implementation of Balzer's algorithm on 28 cells (right).

been assumed to be $\mathrm{O}(1)$-bit data. Here, we introduce a new class of cellular automata for which inter-cell communication is restricted to 1-bit data. A cellular automaton with 1-bit inter-cell communication consists of a onedimensional array of finite state automaton $A=(Q, \delta)$, where

(1) $Q$ is a finite set of internal states.

(2) $\delta$ is a function, defining the next state of any cell and its binary outputs to its left and right neighbor cells, such that $\delta: Q \times\{0,1\} \times\{0,1\} \rightarrow Q \times\{0,1\} \times\{0,1\}$, where $\delta(p, x, y)=\left(q, x^{\prime}, y^{\prime}\right), p, q \in Q, x, x^{\prime}, y, y^{\prime} \in\{0,1\}$, has the following meaning. We assume that at step $t$ the cell $\mathrm{C}_{i}$ is in state $p$ and is receiving binary inputs $x$ and $y$ from its left and right communication links, respectively. Then, at the next step $t+1, \mathrm{C}_{i}$ assumes state $q$ and outputs $x^{\prime}$ and $y^{\prime}$ to its left and right communication links, respectively. Note that binary inputs to $\mathrm{C}_{i}$ at step $t$ are also outputs of $\mathrm{C}_{i-1}$ and $\mathrm{C}_{i+1}$ at step $t$. A quiescent state $q \in Q$ has a property such that $\delta(q, 0,0)=(q, 0,0)$.

$\mathrm{An} \mathrm{O}(1)$-bit $\mathrm{CA}$ is a conventional CA in which the number of communication bits exchanged at one step between neighboring cells is assumed to be $\mathrm{O}(1)$-bit, however, such inter-cell bit-information exchange has been hidden behind the definition of conventional automata-theoretic finite state description. On the other hand, the 1-bit inter-cell communication model is a new $\mathrm{CA}$ in which inter-cell communication is restricted to 1-bit data, referred to as the 1-bit $C A$ model. The number of internal states of the 1-bit CA is assumed to be finite in the usual sense. The next state of each cell is determined by the present state of that cell and two binary 1-bit inputs from its left and right neighbor cells. Thus, the 1-bit CA can be thought of as one of the most powerless and simplest models in a variety of CA's.

Mazoyer [7] and Nishimura, Sogabe and Umeo [11] each designed an optimum-time synchronization algorithm on a 1-bit CA model, based on Balzer's algorithm and Waksman's algorithm, respectively. In Fig. 10, we show a configuration on 25 cells that is based on the 1-bit CA model of Nishimura, Sogabe and Umeo [11]. Each cell has 78 internal states and 208 transition rules. The small black right and left triangles $\boldsymbol{\bullet}$ and $\boldsymbol{\hookrightarrow}$, shown in Fig. 10, indicate a 1bit signal transfer in the right or left direction, respectively, between neighboring cells. A symbol in a cell shows the internal state of the cell. 

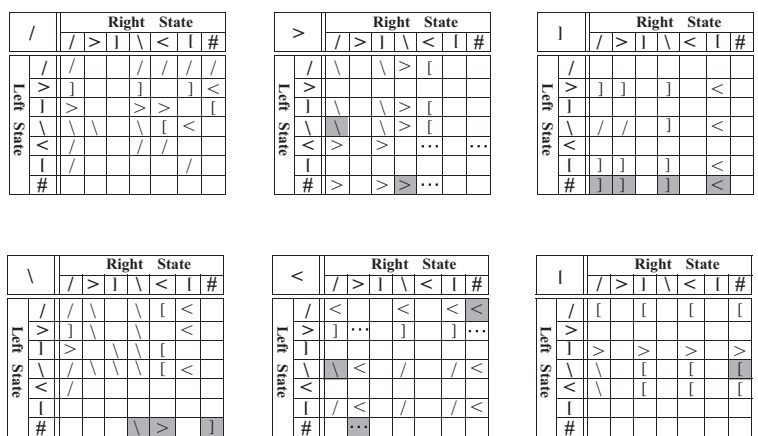

: Rules deleted

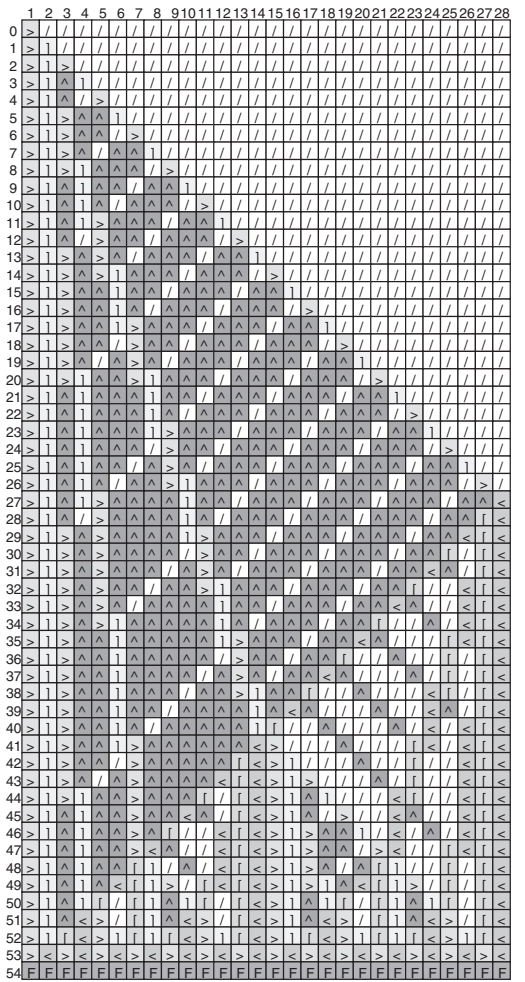

Fig. 6. Transition table for the seven-state Gerken's algorithm (left) and a configuration of the seven-state implementation of Gerken's algorithm on 28 cells (right). In the implementation shown at right, the letter " $F$ " rather than "..." is used to indicates the firing state.
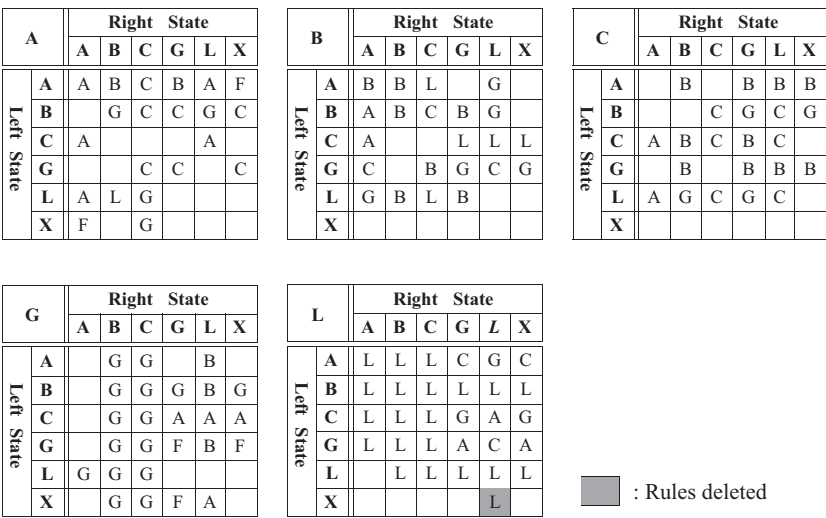

: Rules deleted

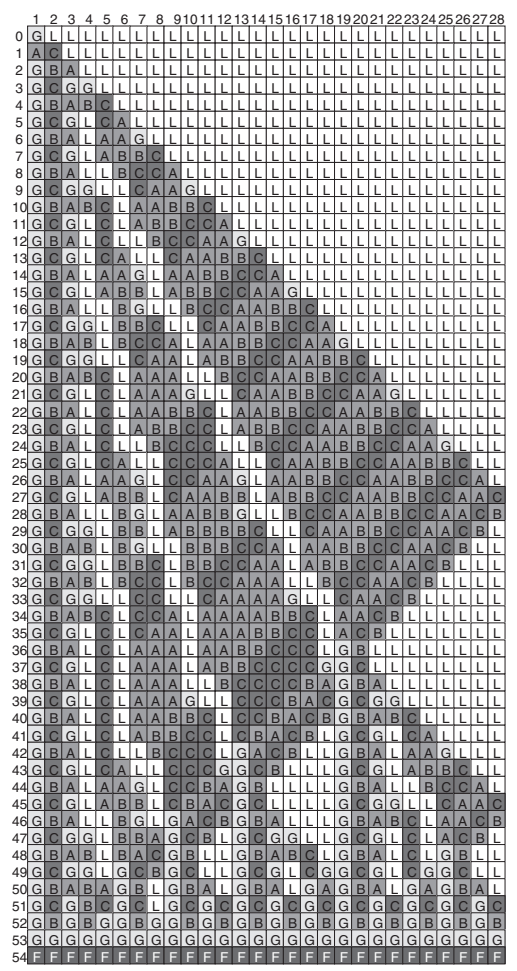

Fig. 7. Transition table for the six-state Mazoyer's algorithm (left) and a configuration of the six-state implementation of Mazoyer's algorithm on 28 cells (right). 


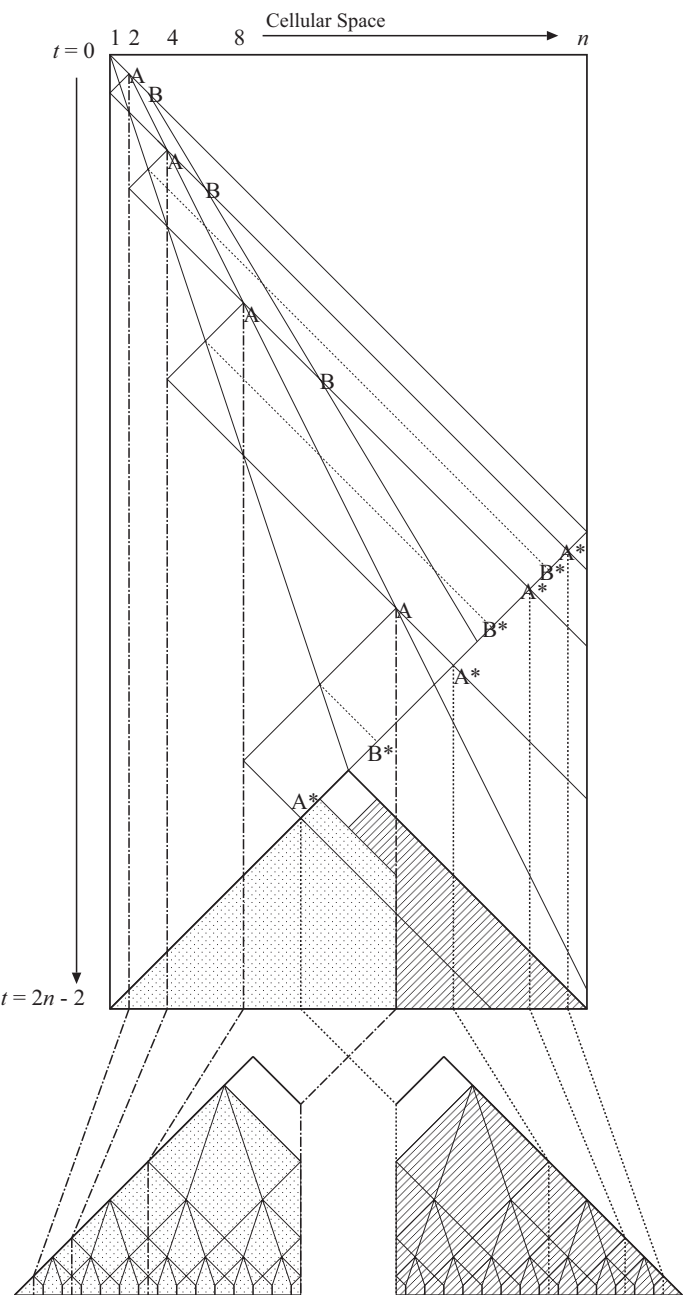

Fig. 8. Time-space diagram for Goto's algorithm as reconstructed by Umeo [12].

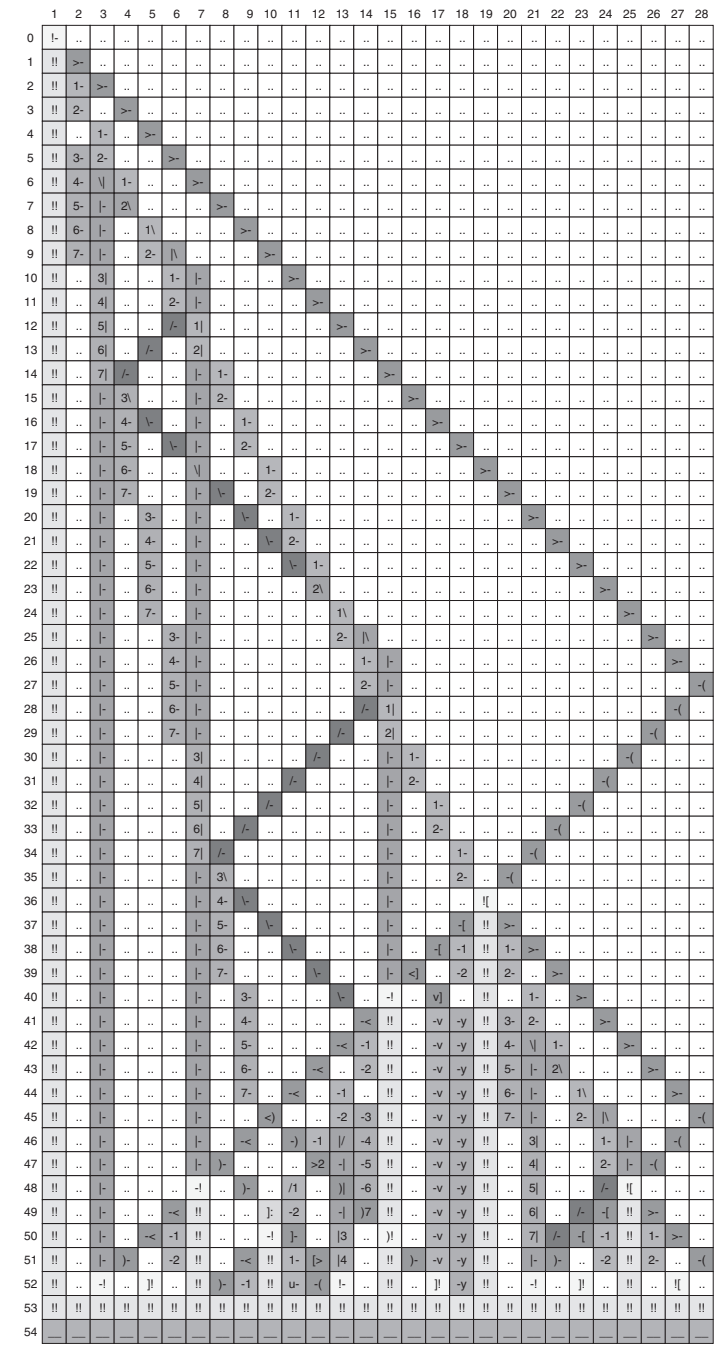

Fig. 9. Configuration of a 155-state implementation of Gerken's algorithm II on 28 cells.

Table 1. Comparison of transition rule sets for optimum-time firing squad synchronization algorithms. The "*”" symbol in parenthesis shows the correction and reduction of transition rules made in this paper. The "**" symbol indicates the number of states and rules obtained after the expansion of the original two-layer construction.

\begin{tabular}{cccc}
\hline Algorithm & \# of states & \# of transition rules & \# of state changes \\
\hline Goto [1962] & many thousands & - & $\mathrm{O}(n \log n)$ \\
Waksman [1966] & 16 & $3216\left(202^{*}\right)$ & $\mathrm{O}\left(n^{2}\right)$ \\
Balzer [1967] & 8 & $182\left(165^{*}\right)$ & $\mathrm{O}\left(n^{2}\right)$ \\
Gerken I [1987] & 7 & $118\left(105^{*}\right)$ & $\mathrm{O}\left(n^{2}\right)$ \\
Mazoyer [1987] & 6 & $120\left(119^{*}\right)$ & $\mathrm{O}\left(n^{2}\right)$ \\
Gerken II [1987] & $32\left(155^{* *}\right)$ & $347\left(2371^{* *}\right)$ & $\mathrm{O}(n \log n)$ \\
\hline
\end{tabular}

\section{Theorem $6^{[7,11,13]}$ There exists a 1-bit CA that can synchronize $n$ cells in optimum $2 n-2$ steps.}

\section{Summary}

Cellular automata researchers have reported that several errors are included in Waksman's transition table. However, the validity of the transition rule sets designed thus far has never been confirmed. This was one of our motivations that we started our study. In the present paper, we have examined via computer the state transition rule sets for which optimum-time synchronization algorithms have been designed over the past forty years. The first transition rule set designed by Waksman [17] includes fundamental errors which cause unsuccessful firings and ninety-three percent of 


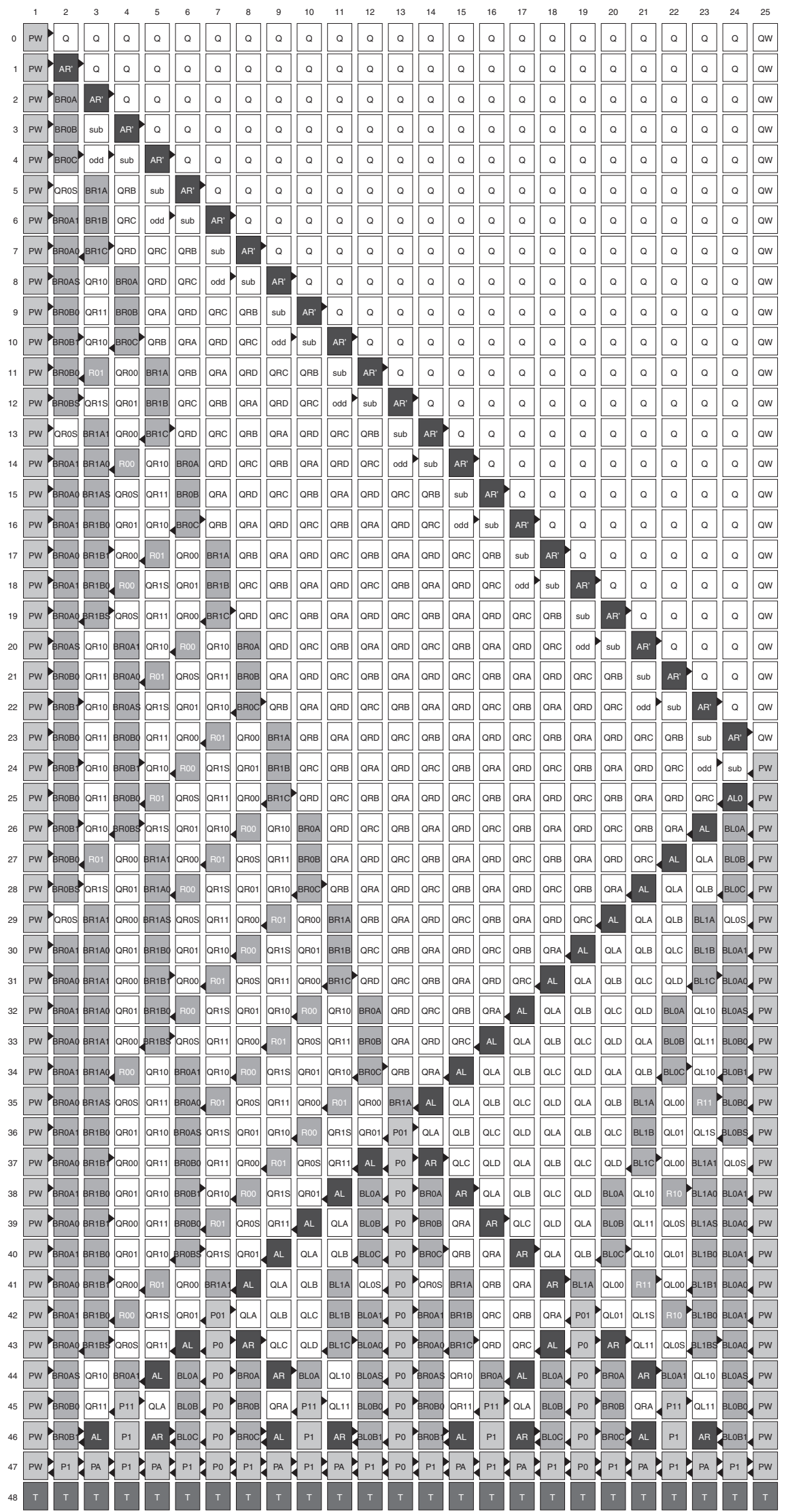

Fig. 10. Configuration of optimum-time synchronization algorithm with 1-bit inter-cell communication on 25 cells. 
the rules are redundant. In addition, the transition rule sets given by Balzer [1], Gerken [2] and Mazoyer [6] also include redundant rules. The authors think that it is worthy of publishing such smallest transition rule sets for the famous firing squad synchronization algorithms, and they are useful and important for researchers who might have interests in those transition rule sets that realize the classical optimum-time firing algorithms quoted frequently in the literatures.

\section{REFERENCES}

[1] Balzer, R., (1967), An 8-state minimal time solution to the firing squad synchronization problem, Inf. Control, $10: 22-42$.

[2] Gerken, Hans-D., (1987), über Synchronisations_-Probleme bei Zellularautomaten, Diplomarbeit, Institut für Theoretische Informatik, Technische Universität Braunschweig, 1-50 (in German).

[3] Goto, E., (1962), A minimal time solution of the firing squad problem, Dittoed Course Notes Appl. Math., Harvard Univ., 298: $52-59$, with an illustration in color.

[4] Goto, E., (1966), "Some puzzles on automata," in Toward computer sciences, ed. by T. Kitagawa, Kyouritsu, Tokyo, 67-91.

[5] Mazoyer, J., (1986), An overview of the firing squad synchronization problem, Lecture Notes Comput. Sci., 316: 82-93.

[6] Mazoyer, J., (1987), A six-state minimal time solution to the firing squad synchronization problem, Theor. Comput. Sci., 50: $183-238$.

[7] Mazoyer, J., (1996), On optimal solutions to the firing squad synchronization problem, Theor. Comput. Sci., 168: 367-404.

[8] Mazoyer, J., (1997), A minimal-time solution to the FSSP without recursive call to itself and with bounded slope of signals, draft version, 8 .

[9] Minsky, M., (1967), Computation: Finite and infinite machines, Prentice Hall, 28-29.

[10] Moore, E. F., (1964), “The firing squad synchronization problem," in Sequential Machines, Selected Papers, ed. by E. F. Moore, Addison-Wesley, Reading, MA, 213-214.

[11] Nishimura, J., Sogabe, T., and Umeo, H., (2000), A design of optimum-time firing squad synchronization algorithm on 1-bit cellular automaton, Tech. Rep. IPSJ, 32-12: 41-44.

[12] Umeo, H., (1996), A note on firing squad synchronization algorithms-A reconstruction of Goto's first-in-the-world optimumtime firing squad synchronization algorithm, Proc. Cellular Automata Workshop, ed. by M. Kutrib and T. Worsche, p. 65.

[13] Umeo, H., Nishimura, J., and Sogabe, T., (2000), 1-bit inter-cell communication cellular algorithms (invited lecture), Proc. Tenth Int. Colloq. Differential Equations, Plovdiv, 1999, Int. J. Diff. Eq. Appl., 1A: 433-446.

[14] Umeo, H., Sogabe, T., and Nomura, Y., (2000), Correction, optimization and verification of transition rule set for Waksman's firing squad synchronization algorithm, Proc. Fourth Int. Conf. Cellular Automata for Research and Industry, Springer, Heidelberg, 152-160.

[15] Vollmar, R., (1979), Algorithmen in Zellularautomaten, Teubner, 192 (in German).

[16] Vollmar, R., (1982), Some remarks about the "Efficiency" of polyautomata, Int. J. Theor. Phys., 21: 1007-1015.

[17] Waksman, A., (1966), An optimum solution to the firing squad synchronization problem, Inf. Control, 9: 66-78. 
